\title{
Deducción de la fórmula de la resistencia esférica en un ensayo experimental
}

Deduction of the spherical resistance formula in an experimental test

Recibido: abril 26 de 2016 | Revisado: agosto 12 de 2016 | Aceptado: setiembre 20 de 2016

\author{
Carlos H.J. Calderón \\ Снамоснumbi $^{\dagger}$ \\ Gian Carlo Scarpati Gálvez ${ }^{\mathrm{I}}$
}

\begin{abstract}
Resumen
El objetivo de esta investigación fue deducir la fórmula de un modelo de resistencia esférica. Para ello, se efectuó el ensayo experimental de un conductor esférico y propuso la implementación de reóstatos esféricos y elipsoidales. Con la metodología establecida surgieron dos dificultades: la primera, en la obtención del material suficientemente grande para la fabricación de la esfera y la segunda, la fragilidad del mismo. Los valores de la resistencia obtenidos en el experimento confirman la validez de los cálculos teóricos con un margen de tolerancia del $20 \%$, totalmente aceptable, considerando las imperfecciones del material utilizado.
\end{abstract}

Palabras clave: resistencia esférica, carbón mineral

\begin{abstract}
The objective of this research was to derive the formula of a spherical resistance model. In order to achieve this, the experimental test of a spherical conductor was carried out and proposed the implementation of spherical and ellipsoidal rheostats. With the methodology established two difficulties arose: the first, obtaining large enough material to manufacture the sphere and second, the fragility of said sphere. The resistance values obtained in the experiment confirm the validity of the theoretical calculations with a tolerance range of $20 \%$, totally acceptable, considering the imperfections of the material used.
\end{abstract}

Key words: spherical strength, mineral coal

1 Universidad de San Martín de Porres Perú gscarpatig@usmp.pe 


\section{Introducción}

En el curso Teoría de Campos de la Escuela Profesional de Ingeniería Electrónica se desarrolla, entre muchos otros tópicos, el modelo de una resistencia rectangular y a partir del mismo se deduce la fórmula de la resistencia de un elemento diferencial de volumen de un conductor. Como aplicaciones estándares se determina la resistencia de un tubo cilíndrico y la de un cascarón esférico, ambos conductores, entre sus caras internas y externas (Stratton, 1941).

Posteriormente, se consultó el valor de la resistencia de una esfera conductora sólida entre sus polos norte y sur. Como el valor de la resistencia resultó ser infinito el problema no fue considerado y nuevamente se replanteó y generalizó para determinar la resistencia entre dos círculos menores paralelos de la esfera sin sus respectivos casquetes esféricos. Adicionalmente, se repitió el cálculo para un conductor elipsoidal (Hayt, 1989).

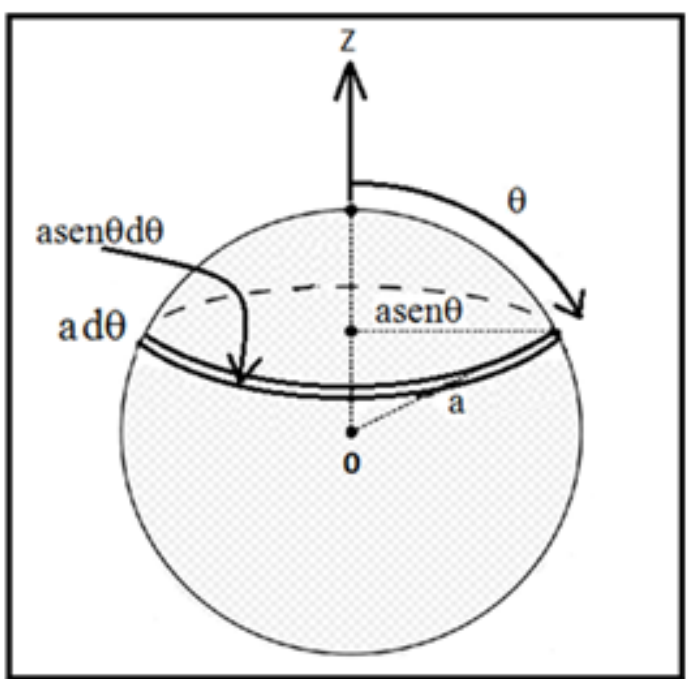

Finalmente, en el laboratorio de electrónica de la Escuela Profesional de Ingeniería Electrónica se adosó bornes para llevar a cabo mediciones de la resistencia de un modelo rectangular de carbón confeccionado para tal fin; así como del modelo esférico fabricado.

La resistencia $\mathrm{d} R$ de un elemento diferencial de columen $\mathrm{d} V$, longitud $\mathrm{d} \ell$ y sección transversal de área $\mathrm{A}$ y conductividad $\sigma_{0}$ es

$$
\mathrm{d} R=\mathrm{d} \ell /\left(\sigma_{0} \mathrm{~A}\right)
$$

Para el caso de una esfera de radio a se tiene

$$
A=\pi a^{2} \operatorname{sen}^{2} \theta, d \ell=a \operatorname{sen} \theta d \theta \quad(2 a, b)
$$

En la Figura 1, se muestra el detalle geométrico necesario para el cálculo del área $y$ del espesor del elemento diferencial de resistencia esférica.

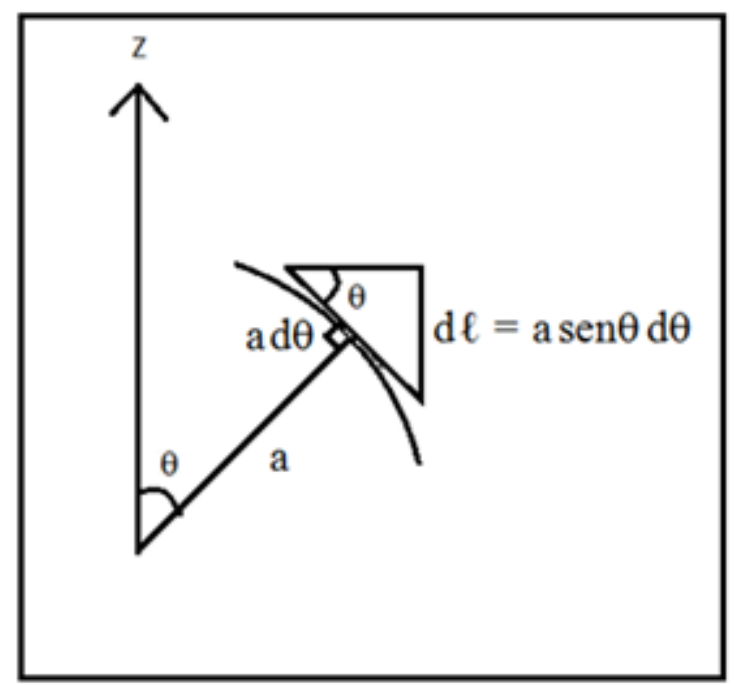

Figura 1. Elemento diferencial de resistencia esférica y detalle del cálculo de $\mathrm{d} \ell$.

Entonces

$$
\begin{gathered}
\mathrm{d} \mathrm{R}=\left[1 /\left(\sigma_{0} \pi \mathrm{a}\right)\right] \mathrm{d} \boldsymbol{\theta} / \operatorname{sen} \boldsymbol{\theta}=\left[1 /\left(\sigma_{0} \pi \mathrm{a}\right)\right] \mathrm{d}[\operatorname{Ln}(\tan \theta / 2)] \\
\mathrm{R}=\left.\left[1 /\left(\sigma_{0} \pi \mathrm{a}\right)\right] \operatorname{Ln}(\tan \theta / 2)_{\theta 0}\right|^{\theta_{1}}=\left[1 /\left(\sigma_{0} \pi \mathrm{a}\right)\right] \operatorname{Ln}\left[\left(\tan \theta_{1} / 2\right) /\left(\tan \theta_{0} / 2\right)\right]
\end{gathered}
$$


Aquí $\theta_{0}$ y $\theta_{1}$ son las distancias polares de los casquetes a ser removidos. Para el caso en que los casquetes sean iguales, es decir,

$$
\theta_{1}=\pi-\theta_{0}
$$

$$
\mathrm{R}=\left[-2 /\left(\sigma_{0} \pi \mathrm{a}\right)\right] \operatorname{Ln}\left(\tan \theta_{0} / 2\right), 0 \leq \theta_{0} \leq \pi / 2
$$

Se observa que dentro del intervalo $0 \leq$ $\theta_{0} \leq \pi / 2$ los valores máximo y mínimo de la resistencia, $R_{M} y R_{m}$, respectivamente, son:

$$
\mathrm{R}_{\mathrm{M}} \rightarrow \infty, \theta_{0}=0, \mathrm{R}_{\mathrm{m}}=0, \theta_{0}=\pi / 2 \quad(7 \mathrm{a}, \mathrm{b})
$$

Derivando la expresión (6) respecto a $\theta_{0}$ rinde

$$
\mathrm{d} R / \mathrm{d} \theta_{0}=-2{ }_{0} /\left(\sigma_{0} \pi \mathrm{a} \operatorname{sen} \theta_{0}\right)
$$

Dentro del intervalo $0 \leq \theta_{0} \leq \pi / 2$ la expresión (8) rinde valores negativos mono tónicamente crecientes así que

$$
0 \leq \mathrm{R}<\infty
$$

En la Figura 2, se presenta la variación del valor de la resistencia normalizada, $\mathrm{R}_{\mathrm{n}}$, respecto a $2 /\left(\pi \mathrm{a} \sigma_{0}\right)$ entre dos círculos menores paralelos e iguales de una esfera conductora en función de la distancia polar $\boldsymbol{\theta}_{0}$.

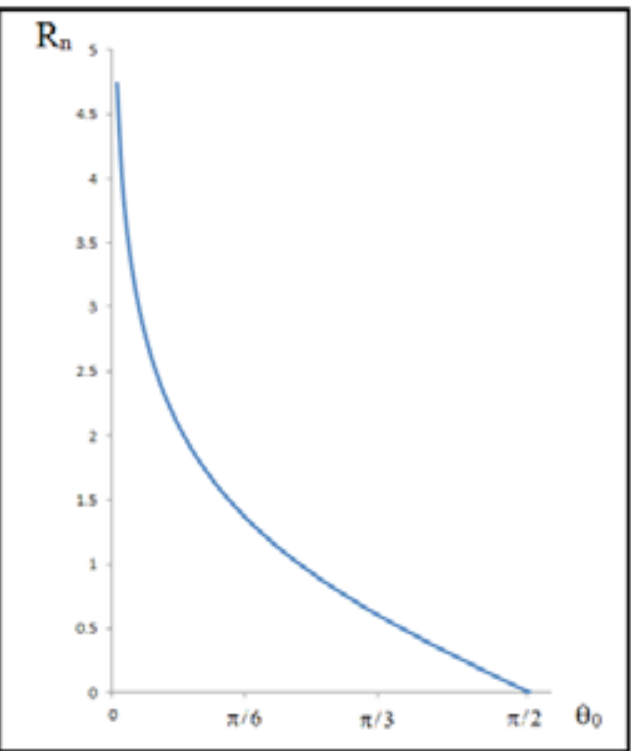

Figura 2. Resistencia normalizada entre dos círculos menores paralelos e iguales de una esfera conductora en función de la distancia polar $\theta_{0}$.
Para la determinación del campo escalar del potencial eléctrico se eligió la solución trivial de la ecuación de Laplace en coordenadas esféricas (Magid, 1950) es decir,

$$
\Phi=(\mathrm{A}+\mathrm{B} / \mathrm{r})[\mathrm{C}+\mathrm{D} \operatorname{Ln}(\tan \theta / 2)](\mathrm{E}+\mathrm{F} \varphi)
$$

Además, sujeta a la condiciones de frontera:

$$
\text { (1) } \Phi=+\mathrm{V}_{0}, \boldsymbol{\theta}=\boldsymbol{\theta}_{0} \text {; (2) } \Phi=-\mathrm{V}_{0}, \boldsymbol{\theta}=\pi-\boldsymbol{\theta}_{0} \text {; }
$$$$
\text { (3) } \Phi=0, \theta=\pi / 2
$$

Dada la simetría axial del problema la solución debe ser independiente del acimut. Por tanto:

$$
\Phi=(\mathrm{A}+\mathrm{B} / \mathrm{r})[\mathrm{C}+\mathrm{D} \operatorname{Ln}(\tan \theta / 2)] \mathrm{E}
$$

La aplicación de la condición de frontera (1) deviene en:

$$
\mathrm{V}_{0}=(\mathrm{A}+\mathrm{B} / \mathrm{r})\left[\mathrm{C}+\mathrm{D} \operatorname{Ln}\left(\tan \theta_{0} / 2\right)\right] \mathrm{E}
$$

Como la condición debe ser cumplida para $\forall \mathrm{r}$ en $0 \leq \mathrm{r} \leq \mathrm{a}$ se deduce que $\mathrm{B}=0$ así que

$$
\Phi=\mathrm{A}[\mathrm{C}+\mathrm{D} \operatorname{Ln}(\tan \theta / 2)] \mathrm{E}
$$

La imposición de la condición (3) exige que

$$
0=\mathrm{A}[\mathrm{C}+\mathrm{D} \operatorname{Ln}(\tan \pi / 4)] \mathrm{E}=\mathrm{A} \mathrm{CE} \quad(14
$$

Entonces,

$$
\begin{gathered}
C=0 \\
\Phi=\mathrm{A} D E \operatorname{Ln}(\tan \theta / 2)
\end{gathered}
$$

Aplicando la condición (2) da

$$
\begin{gathered}
\mathrm{ADE}=\mathrm{V}_{0} / \operatorname{Ln}\left(\tan \theta_{0} / 2\right) \\
\Phi=\left[\mathrm{V}_{0} / \operatorname{Ln}\left(\tan \theta_{0} / 2\right)\right] \operatorname{Ln}(\tan \theta / 2)
\end{gathered}
$$


Entonces

$$
\begin{gathered}
\mathbf{E}=-\mathbf{u}_{\theta}\left[\mathrm{V}_{0} / \operatorname{Ln}\left(\tan \theta_{0} / 2\right)\right] /(\mathrm{r} \operatorname{sen} \boldsymbol{\theta}) \\
\mathbf{J}=-\mathbf{u}_{\theta}\left[\sigma_{0} \mathrm{~V}_{0} / \operatorname{Ln}\left(\tan \theta_{0} / 2\right)\right] /(\mathrm{r} \operatorname{sen} \theta)
\end{gathered}
$$

El elemento diferencial de área $\mathrm{d} \mathbf{A}_{\theta}$ viene dado por

La corriente total $\mathrm{I}_{0}$ es

$$
\mathrm{d} \mathbf{A}_{\theta}=\mathbf{u}_{\theta} \mathrm{r} \operatorname{sen} \theta \mathrm{d} \varphi \mathrm{d} \mathrm{r}
$$

$$
\begin{gathered}
\mathrm{I}_{0}={ }_{0} \int{ }_{0}^{2 \pi} \int \mathrm{a} \mathbf{J} \cdot \mathrm{d} \mathbf{A}_{\theta}=-{ }_{0} \int 2 \pi{ }_{0} \int \mathrm{a}\left[\sigma_{0} \mathrm{~V}_{0} / \operatorname{Ln}\left(\tan \theta_{0} / 2\right)\right] \mathrm{d} \varphi \mathrm{d} \mathrm{r}=-2 \pi \mathrm{a} \sigma_{0} \mathrm{~V}_{0} / \operatorname{Ln}\left(\tan \theta_{0} / 2\right) \\
\mathrm{R}_{\mathrm{C}}=2 \mathrm{~V}_{0} / \mathrm{I}_{0}=\left[-1 /\left(\pi \mathrm{a} \sigma_{0}\right)\right] \operatorname{Ln}\left(\tan \theta_{0} / 2\right)
\end{gathered}
$$

Esta resistencia corresponde a la de solo un hemisferio porque el plano ecuatorial de la esfera está puesto a tierra así que

$$
\mathrm{R}=2 \mathrm{R}_{\mathrm{C}}=\left[-2 /\left(\pi \mathrm{a} \sigma_{0}\right)\right] \operatorname{Ln}\left(\tan \theta_{0} / 2\right)
$$

En consecuencia, tanto el cálculo directo como el que sigue la vía de los campos electrostáticos rinden el mismo valor de la resistencia entre dos círculos menores paralelos e iguales de una esfera conductora en función de la distancia polar $\theta_{0}$.

La potencia consumida al aplicar un potencial eléctrico $\mathrm{E}$ a través de los terminales de la resistencia esférica $R$ es

${ }_{\mathrm{i}} \mathrm{P}=\mathrm{E}^{2} / \mathrm{R}=-\pi \mathrm{a} \sigma_{0} \mathrm{E}^{2} /\left[2 \operatorname{Ln}\left(\tan \theta_{0} / 2\right)\right]$

Utilizando el equivalente mecánico del calor, $\mathrm{J}=4.1855 \mathrm{~J} / \mathrm{cal}$, en conjunción con las características térmicas del material conductor utilizado es posible determinar tanto la temperatura como el volumen de trabajo de la resistencia.

\section{Método}

El primer paso fue determinar el material con el cual implementar el prototipo desarrollado. Dicho material debía ofrecer determinadas características físicas, tanto en lo relativo a su resistividad como a la facilidad de obtenerlo y además debía tenerse en cuenta la dificultad para darle la forma esférica requerida. Luego de realizar diferentes pruebas con distintos materiales, se eligió al carbón mineral pues cumplía razonablemente con las características requeridas. El carbón mineral es una roca sedimentaria de color negro, muy rica en carbono y con cantidades variables de otros elementos, principalmente hidrógeno, azufre, oxígeno y nitrógeno (Danus, 2010).

Con la finalidad de determinar la resistividad de la muestra se cortó manualmente un especimen rectangular con longitud de $47 \mathrm{~mm}$ y sección transversal de $10 \mathrm{~mm} \times 10$ $m m$. Para el efecto se utilizó una hoja de sierra HSS (High Speed Steel). El especimen se ilustra en la Figura 5. Para el resistor esférico de radio $45.3 \mathrm{~mm}$, se usó un torno. El corte de los casquetes polares, a un ángulo de 37 o se realizó también, manualmente, con una hoja de sierra del mismo tipo. El resistor esférico se ilustra en la Figura 4.

Para efectuar las mediciones de resistencia, a ambas muestras se les sujetó con placas metálicas a guisa de bornes en los extremos de cada pieza (Figuras 4 y 5). Se utilizó un multímetro marca SIMPSON, modelo 260, a una temperatura ambiente de aproximadamente $20^{\circ} \mathrm{C}$. La resistencia de los cables conductores y los bornes se midió poniendo 
en cortocircuito a los bornes cuyo valor resultante fue restado de las respectivas resistencias.

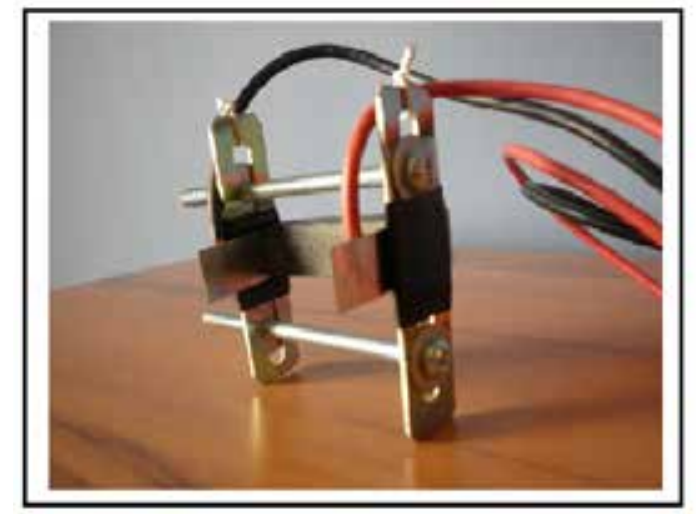

Figura 3. Fotografía del modelo rectangular.

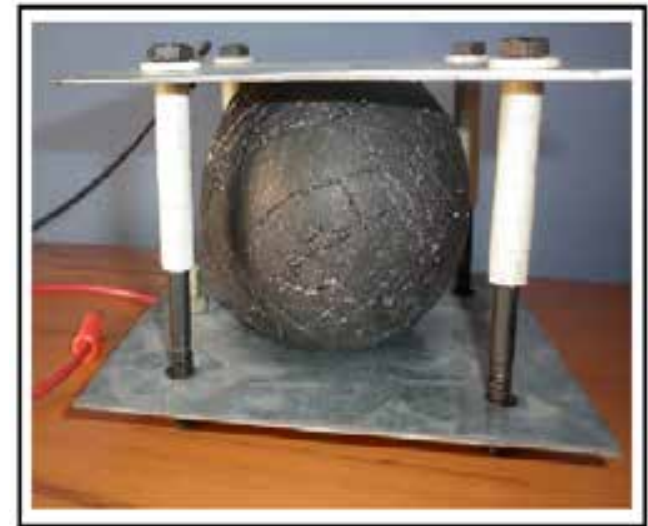

Figura 4. Fotografía de la resistencia esférica

\section{Resultados}

La resistencia de los cables conductores y los bornes fue de $0.5 \Omega$ y la resistencia conjunta fue de $15.6 \Omega$ así que la resistencia del espécimen fue de $15.1 \Omega$.

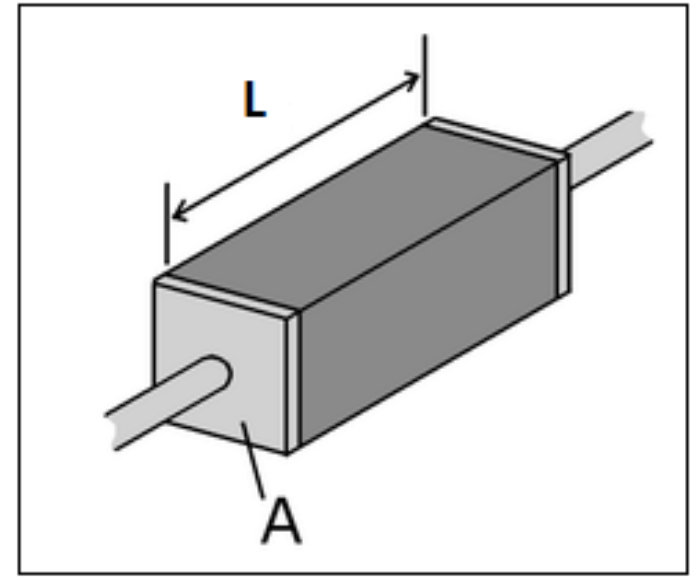

Figura 5. Modelo rectangular de carbón.
La resistividad $\rho_{0}$, inversa de la conductividad $\sigma_{0}$, viene dada por

$$
\rho_{0}=\mathrm{R} \times \mathrm{A} / \mathrm{L} \Omega m
$$

Entonces

$$
\rho_{0}=32.13 \times 10^{-3} \Omega m
$$

Por lo tanto,

$$
\sigma_{0}=31.12 \Omega^{-1} / m
$$

En el caso de la esfera, la resistencia combinada de los cables conductores y los bornes fue de $1.9 \Omega$ y la resistencia conjunta fue de $2.4 \Omega$; así que la resistencia del resistor esférico fue de $0.5 \Omega$.

La primera dificultad consistió en obtener el material lo suficientemente grande para la fabricación de la esfera, pues se necesitaba que la muestra fuera de dimensiones mínimas para poder ser trabajada. La segunda dificultad que se tuvo al dar forma a los modelos pues el material, por sus características físicas, tiende a astillarse y romperse con facilidad, demandando un cuidado extremo al momento de trabajarse.

\section{Conclusiones}

Los valores de las resistencias obtenidos experimentalmente confirman la validez de los cálculos teóricos dentro de un margen de tolerancia del 20\% totalmente aceptable considerando las imperfecciones del material utilizado.

\section{Recomendaciones}

Se recomienda que en una segunda etapa, se considere el diseño e implementación de un reóstato elipsoidal utilizando carbono pulverizado y compactado con algún tipo de molde. Además se recomienda optimizar el dominio de operación y estudiar la corrosión progresiva entre los bornes y las muestras sugeridas por el exprofesor Juan Francisco Paredes Lozano. 


\section{Agradecimiento}

Los autores dejan constancia de su agradecimiento a las autoridades académicas de la Universidad de San Martín de Porres y a las de la Facultad de Ingeniería y Arquitectura, en particular, por propiciar las condiciones de trabajo requeridas para la investigación científica. De manera específica, desean agradecer al señor profesor Ing. don Rafael Figueroa Lezama por su excelente solvencia profesional y su gran vocación de servicio al fabricar el conductor esférico en su propio taller a partir de un trozo de grafito que le fue proporcionado.

\section{Referencias}

Danus, H. (2010). Carbón, protagonista del pasado, presente y futuro. Chile: RIL Editores.

Hayt, W. (1989). Engineering Electromagnetics. New York: McGraw-Hill.
Magid, L. (1950). Electromagnetic Fields, Energy, and Waves. New York: John Wiley $\&$ Sons, Inc.

Stratton, J.A. (1941). Electromagnetic theory. New York: McGraw-Hill. 